\title{
Breastfeeding - the best diet and health care measure
}

\section{Adrian Balanean}

Scientific Manager Nestle Infant Nutrition Romania

Human milk is a highly complex and dynamic mixture of numerous essential ingredients. Their specific combination enables newborns to grow healthily. Compared to non-breastfed children, breastfed infants suffer fewer and shorter infections, have different growth patterns and different gut microbiota, undergo better cognitive development, and probably also have a lower risk of chronic diseases such as obesity and cardiovascular disease in later life.

We know that growth in the first years of life will exert a positive influence on long-term health and neurological development. Protein is one important factor in this growth process. The infant's growth velocity decreases rapidly during the first months of life and afterwards slowly continues to decrease along with protein requirements for growth. Both the quantity and quality of protein in human milk change continuously over time. Trials suggest that infant formulas containing less (protein closer to the protein content in breast milk), but of the right quality, can support weight gain closer to that undergone by breastfed infants.

On the next few pages, I would like to give you a brief overview of the scientific status quo and to present a few additional expert opinions to illustrate the current discussion.

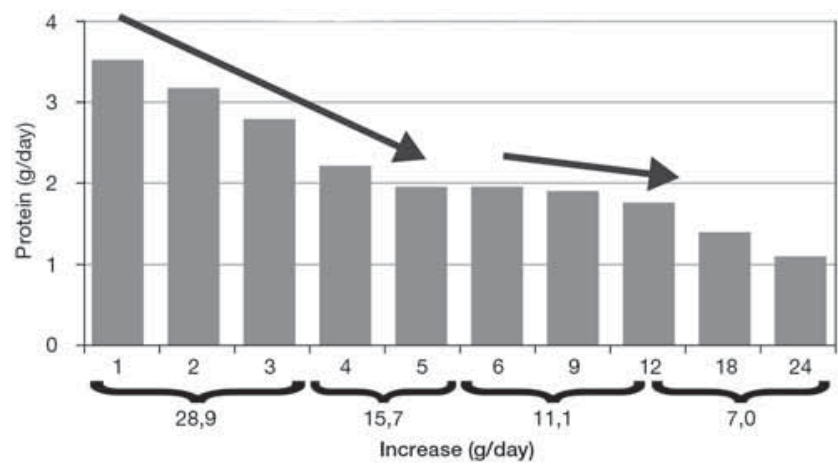

FIGURE 1. Protein requirements for growth

Adapted from Fomon et al. AJCN 1982
Mother's own milk is the best source of nutrition for all healthy infants (Martin et al. 2016). The often used slogan "breast is best" is an oversimplification of the benefits of breastfeeding (ESPGHAN 2009). Breastfed infants experience fewer and shorter infections, have different growth patterns, different gut microbiota, better cognitive development and even probably a lower risk of chronic diseases such as obesity and cardiovascular disease in later life compared to non-breastfed children (Lonnerdal et al. 2016; Lonnerdal et Hernell 2016; WHO 2015). The promotion and protection of breastfeeding are therefore important measures for health prevention.

The CHOP (European Child Obesity Project) study, including healthy term infants from 5 European countries between 2002 and 2004 showed breastfeeding duration far from the recommendations and complementary foods introduced earlier than recommended in a sizeable number of infants (Schiess et al. 2010). In the EU, Breastfeeding experts, interest groups and mothers came together in 2004 to establish an action plan to promote breastfeeding in Europe (EU Project on Breastfeeding). According to a 2015 WHO report, Europe still has the lowest rates of breastfeeding of all the WHO regions 10 years later, although with significant differences within the European region.

The rate of early initiation of breastfeeding is very high in some European countries (WHO 2015). It is mostly the duration of exclusive breastfeeding to 6 months as recommended by the WHO and the continued breastfeeding after the introduction of solid foods that need to be improved. The information and education of expectant and new parents on the health benefits of breastfeeding for mother and child are effective measures for the promotion of breastfeeding that can be easily implemented. It is important to include mothers with lower socioeconomic status (less income, educa- 
tion and employment), who are less likely to begin breastfeeding.

\section{Protein in human milk adapts to the decreasing protein requirements of the infant associated with decreasing growth velocity.}

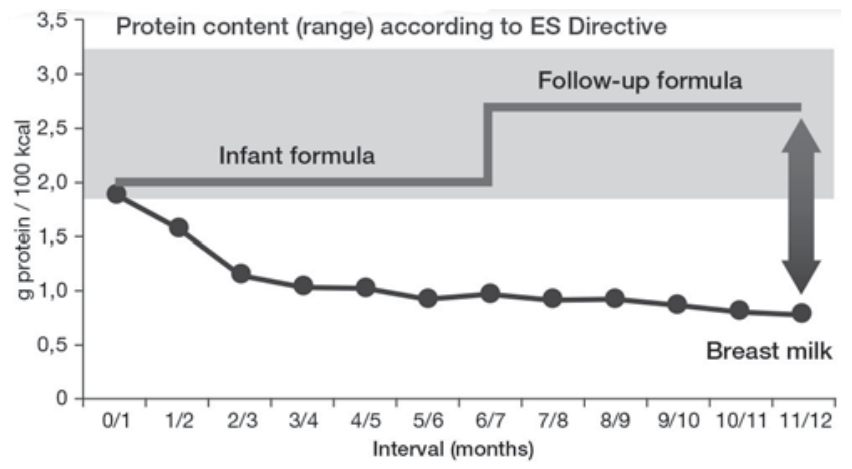

FIGURE 2. Protein content in human milk and current formulas

The infant's growth velocity decreases rapidly in the first months of life and then gradually continues to do so along with protein requirements for growth (Fig. 1).

Protein in breast milk adapts to the changing requirements for growth by rapidly decreasing in the first months of life and then gradually continuing to do so (Fig. 2). In the current formula stage systems the reverse is the case: the protein content in followup formulas is higher than that in starter formulas, so that the difference between the protein content in formula and human milk becomes continually greater (Fig. 2).

\section{Non-breastfed infants also have a right to a healthy diet}

Even with optimal promotion of breastfeeding, there will always be infants who depend on a substitute for breast milk because their mother cannot or chooses not to breastfeed. Along with the promotion of breastfeeding, it is therefore important to optimize the nutrition of infants needing an infant formula.

\section{Human milk - the gold standard}

The composition of the milk of healthy, wellnourished women is the model for the nutrient composition of formulas. The actual quality of an infant formula can, however, only be determined by comparing its effects on growth, plasma markers and functional outcomes (e.g. immune responses and later health) to those found in populations of healthy exclusively breast-fed infants (ESPGHAN. Koletzko et al. JPGN 2005).

\section{Breastfed infants have different growth patterns and a lower risk of obesity in later life compared to formula-fed infants.}

Growth and development are central characteristics of childhood. The effect of human milk on growth has recently moved into science's focus driven by the worldwide increase of overweight and obesity, also in children. According to estimates from the WHO's Childhood Obesity Surveillance Initiative (COSI), around 1 in 3 children in the EU aged 6-9 years old were overweight or obese in 2015. This is a worrying increase on 2008, when estimates were 1 in 4 (EU Action Plan 2014).

Research shows that breastfed children have a lower risk of obesity in later life compared to formula-fed infants (Arenz et al. 2004; Harder et al. 2005; Horta et al. 2007; Owen et al. 2005; von Kries et al. 1999; Weng et al. 2012, Patro-Golab et al. 2016 ). A meta-analysis based on 113 studies showed that longer periods of breastfeeding were associated with a $26 \%$ reduction in the risk (odds ratio) of overweight or obesity (Horta et al. Acta Paediatr 2015, cited by Victora et al. Lancet 2016). Among the 11 high-quality studies identified in this systematic review, the association between breastfeeding and later overweight/obesity was smaller (13\% risk reduction), but remained significant.

,, The prevention of obesity is especially important, as overweight and obesity are epidemic and therapeutic interventions have shown limited success. In infancy, breastfeeding plays a central role in the prevention of overweight and obesity."

\section{Martin Wabitsch}

Professor, Head of Division of Pediatric Endocrinology and Diabetes and of Department of Pediatrics and Adolescent Medicine, University Medical Center Ulm, Germany.

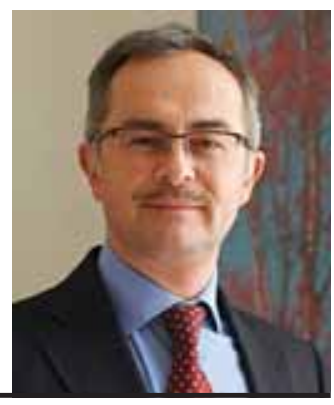

Observational studies have shown that rapid weight gain, defined as weight gain in excess of the median by more than 0.67 standard deviations, i.e. an upward centile crossing through at least one of the major centile bands, in the first two years of life is associated with a two- to threefold increase in 
overweight or obesity risk in childhood, youth and adulthood (Baird et al. 2005; Monteiro et al. 2005; Ong et al., 2006). The workgroup around Koletzko et al. hypothesized that breastfeeding protects by reducing weight gain velocity during early childhood because of its different substrate supply with breastfeeding compared to feeding conventional infant formula, in particular the significantly lower protein content in human milk.

Compared with breastfeeding, feeding conventional formulas is also associated with altered body composition. A systematic review and meta-analysis of 15 studies that examined body composition in healthy infants showed that breast-fed infants had a lower fat mass at 1 year of age than formulafed infants (Gale et al. 2012).

\section{Protein - the major modulator of early growth}

"There is no doubt that early nutrition has a programming effect on an infant's growth and development, as well as long-term health. A wealth of new evidence support the hypothesis of an association of high protein intake in early childhood and increased risk of non-communicable diseases later in life. Using low-protein formula with high protein quality in infants who are not breastfed might be one of the best ways to provide them an opportunity of developing an appropriate metabolic programming."

\section{Natalia Migacheva}

Associate Professor, Department of Pediatrics, Deputy Director of the Institute of Professional Education, Samara State Medical University, Russia

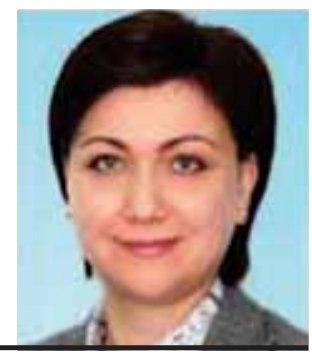

By optimizing protein quality and reducing protein quantity in infant formula, we can achieve an approximation of early growth to that of breastfed infants. The results of a multicenter pooled analysis of individual participant data $(\mathrm{n}=1882)$ from 11 randomized controlled trials (Alexander et al. 2016) shows this: The weight-for-age z-scores at 4 months of age of healthy term infants fed wheypredominant formulas with $1.8 \mathrm{~g}$ protein $/ 100 \mathrm{kcal}$ (lower content than in most current formulas and closer to breast milk) and improved quality from - 4 weeks of age to - 4 months of age were very close to the mean WHO standard (zscore 0) and the 95\% confidence intervals were within $\pm 0.5 \mathrm{SD}$ (Standard Deviation) of WHO growth standards.

The effect of early protein intake on growth was tested in a large multi-country study, the European Childhood Obesity Program (CHOP) (Koletzko et al. 2009; Koletzko et al. 2014). Formula fed infants were randomly assigned to lower or higher protein formulas (infant formula with $1.8 \mathrm{~g}$ and $2.2 \mathrm{~g} / 100$ kcal respectively, for the first 4 months and then follow-up formula with $2.9 \mathrm{~g}$ and $4.4 \mathrm{~g} / 100 \mathrm{kcal}$ respectively, until the end of the first year of life Koletzko et al. 2009). Those in the group receiving the formulas with the higher protein content had significantly higher weight gain and, at the ages of 12 and 24 months, BMIs were significantly higher in the higher protein group, even though the intervention stopped at 12 months of age (Koletzko et al. 2009).

Follow-up to school age demonstrated lasting significant effects of early protein supply (Koletzko et al. 2014). At 6 years of age, previously breastfed infants had a significantly lower BMI than those in the control group fed higher protein formulas (Weber et al. 2014). The children fed lower protein formula in the first year of life had a BMI that was significantly lower than the control group and not different from the breastfed reference group.

\section{Formulas with high protein content increase obesity risk at school age}

In the CHOP-study a diet of higher protein formulas during the first year of life increased the risk of being obese at 6 years of age 2.43-fold (unadjusted) and 2.87-fold (adjusted) respectively, compared to a diet of lower protein $(1.8 \mathrm{~g}$ in infant and $2.2 \mathrm{~g} / 100 \mathrm{kcal}$ in follow-up formula) formulas (Weber et al. 2014).

The measurements of pre-peritoneal (PP) fat, a marker of abdominal fat, in a subgroup of children participating in the CHOP study at the age of 5 years, are also interesting.

Subcutaneous fat was not different between the groups, but the PP fat layer was significantly thicker in the higher compared to the lower protein formula group (Cruszfeld et al. 2016). A thicker layer of PP fat may trigger adverse metabolic and health consequences.

This observed long-term effect on later abdominal fat distribution supports the hypothesis that a high protein intake in infancy may increase the risk for cardiovascular diseases (Cruszfeld et al. 2016). 


\section{Does high protein intake during the second 6 months of life and beyond matter?}

In late infancy and among toddlers, the average protein intake is 3 to 4 times as high as the physiological requirements (Fantino et al. 2008; Hilbig et al. 2006; Hornell et al. 2013; Michaelsen et al. 2014).

Several observational studies report a positive correlation between the protein intake in later infancy and the second year of life and later obesity (Rolland-Cachera et al., 1995; Scaglioni et al. 2000). There are indications that a high dairy protein intake especially has an adverse effect on later body composition. One study, for example, shows that dairy, but not meat or cereal protein intake at 12 months is related to body fat percentages at 7 years of age (Günther et al. 2007). A current study showed that an extra $10 \mathrm{~g}$ of protein/day at the age of 12 months was associated with a higher BMI due to a higher fat mass index (FMI), but not with fatfree mass index, at the age of 6 years

(Voortman et al. 2016). The association of protein intake with FMI was stronger for the intake of animal protein (both dairy and non-dairy protein) than protein from vegetable sources. Meat may have a high protein content, but the small amounts of meat needed to ensure good iron status have a lesser impact on total protein intake (Michaelsen et al. 2014). High protein formulas and cow's milk on the other hand can contribute significantly to excessive protein intake.

Taking into account the emerging evidence that high protein intake during the first 2 years of life is a risk factor for the subsequent development of overweight and obesity, it seems prudent to avoid a high protein intake in excess of metabolic requirements during the first 2 years of life (Michaelsen et al. 2014). This could be accomplished with a dynamic formula- stage-system in which protein content and quality reflect changes in human milk and the changing requirements of the infant.

"Although all components of nutrition are relevant for a healthy balanced diet, there is overwhelming evidence that quantity and quality of protein play a key role. "

\section{Wan Vandenplas}

Professor, Head of Department of Paediatrics, UniversitairZiekenhuis Brussels, Belgium

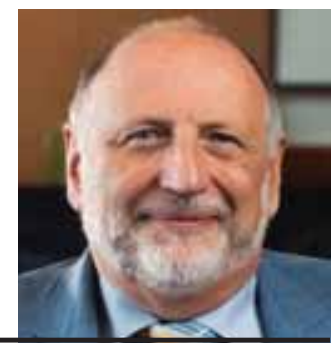

Conflict of interest: none declared

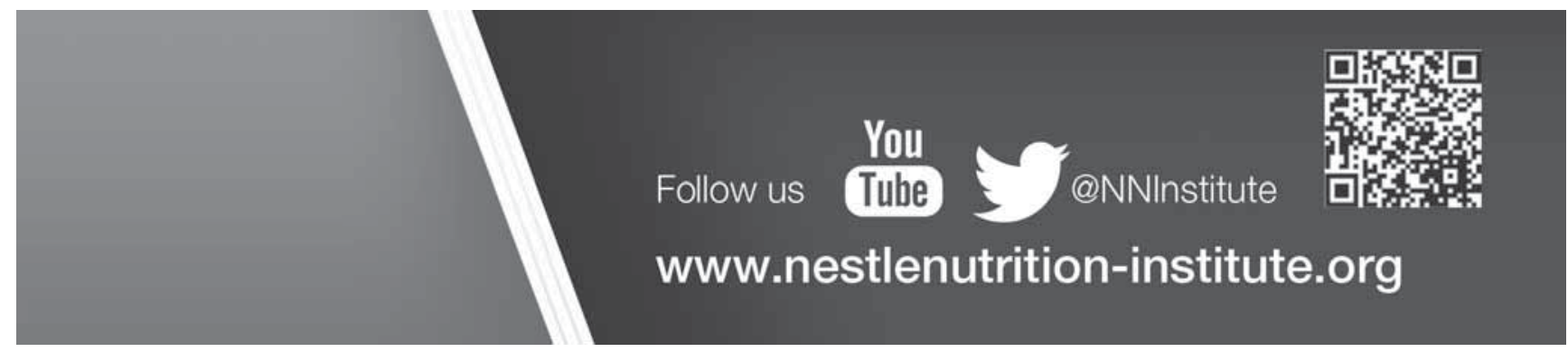

